\title{
Determination of Penetration Depth of Transverse Spin Current in Ferromagnetic Metals by Spin Pumping
}

\author{
Tomohiro Taniguchi ${ }^{1,2}$, Satoshi Yakata ${ }^{3}$, Hiroshi Imamura ${ }^{2}$, Yasuo Ando $^{3}$ \\ ${ }^{1}$ Institute for Materials Research, Tohoku University, Sendai 980-8577, \\ ${ }^{2}$ Nanotechnology Research Institute, National Institute of Advanced Industrial Science and Technology, \\ 1-1-1 Umezono, Tsukuba, Ibaraki 305-8568, Japan, \\ ${ }^{3}$ Department of Applied Physics, Graduate School of Engineering, Tohoku University, Sendai
}

(Dated: November 10, 2018)

\begin{abstract}
Spin pumping in nonmagnetic/ferromagnetic metal multilayers is studied both theoretically and experimentally. We show that the line widths of the ferromagnetic resonance (FMR) spectrum depend on the thickness of the ferromagnetic metal layers, which must not be in resonance with the oscillating magnetic field. We also show that the penetration depths of the transverse spin current in ferromagnetic metals can be determined by analyzing the line widths of their FMR spectra. The obtained penetration depths in NiFe, CoFe and $\mathrm{CoFeB}$ were $3.7[\mathrm{~nm}], 2.5[\mathrm{~nm}]$ and 12.0 [nm], respectively.
\end{abstract}

PACS numbers: 72.25.Mk, 75.70.Cn, 76.50.+g, 76.60.Es

The field of current-driven magnetization dynamics (CDMD) has drawn enormous attention because of its potential applications to non-volatile magnetic random access memory and microwave devices. CDMD is also important from a scientific point of view since it provides much information about non-equilibrium dynamics of the magnetization and physics of spin transport and spin relaxation. The concept of CDMD was first proposed by Slonczewski [1] and independently by Berger 2] in 1996. In the last decade much effort has been devoted to studying the physics and applications of CDMD both theoretically and experimentally $[\underline{3}, 4]$.

One of the most important quantities in CDMD is the penetration depth of the transverse spin current $\lambda_{t}$, over which spin transfer torque is exerted for the magnetization of the free layer. However, there is a controversial issue regarding the penetration depth of the transverse spin current. One argument is based on the ballistic theory of electron transport, and its $\lambda_{\mathrm{t}}=\pi /\left|k_{\mathrm{F}}^{\uparrow}-k_{\mathrm{F}}^{\downarrow}\right|$, which is on the order of the lattice constant in conventional ferromagnets such as $\mathrm{Fe}, \mathrm{Co}, \mathrm{Ni}$, and their alloys [5, 6]. The other argument is based on the Boltzmann theory of electron transport, and its $\lambda_{\mathrm{t}}$ is on the order of a few $\mathrm{nm}$ [7]. Urazhdin et al. analyzed the CPP-GMR of noncollinear magnetic multilayers using the extended twoseries-resistance model and concluded that $\lambda_{\mathrm{t}}=0.8[\mathrm{~nm}]$ for permalloy [8]. On the other hand, Chen et al. analyzed the critical current of the CDMD in the $\mathrm{Co} / \mathrm{Cu} / \mathrm{Co}$ trilayer system and concluded that $\lambda_{\mathrm{t}}=3.0[\mathrm{~nm}]$ for Co [9].

The inverse process of CDMD is spin pumping, where spin current is generated by precession of magnetization in the ferromagnetic layer [10]. Enhancement of the Gilbert damping constant due to spin pumping has been extensively studied, and spin diffusion lengths, i.e., penetration depths of spin current in nonmagnetic metals, have been obtained by analyzing the dependence of the enhancement of the Gilbert damping constant on the thickness of the nonmagnetic metal layer. In spin

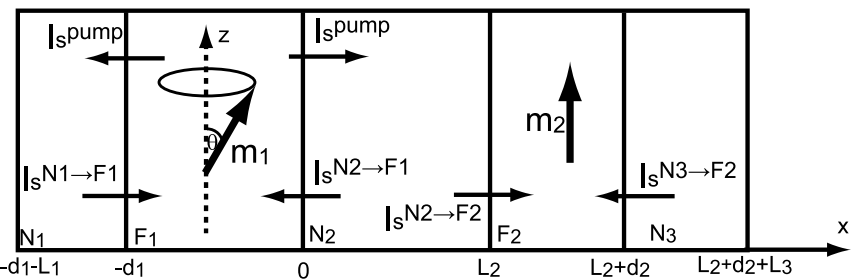

FIG. 1: Schematic illustration of a nonmagnetic/ferromagnetic metal five-layer system. The magnetization of the $\mathrm{F}_{1}$ layer $\left(\mathbf{m}_{1}\right)$ precesses around the $z$-axis with angle $\theta$. The magnetization of the $\mathrm{F}_{2}$ layer $\left(\mathbf{m}_{2}\right)$ is fixed with the $z$-axis. The precession of the magnetization in the $F_{1}$ layer pumps the spin current $\mathbf{I}_{s}^{\text {pump }}$. The pumped spin current creates spin accumulation in the other layers, and the spin accumulation induces a backflow of spin current $\mathbf{I}_{s}^{\mathrm{N} \rightarrow \mathrm{F}}$ across each N/F interface.

pumping, the direction of the magnetization vector of the pumped spin current is perpendicular to the direction of the precessing magnetization vector [10]. Let us consider the nonmagnetic/ferromagnetic metal five-layer system shown in Fig. 11 Since the magnetization vector of the pumped spin current $\mathbf{I}_{s}^{\text {pump }}$ is perpendicular to the magnetization vector $\mathbf{m}_{1}$ of the $\mathrm{F}_{1}$ layer and the precession angle $\theta$ is very small (about 1 [deg]) in conventional FMR experiments, the dominant component of the pumped spin current is perpendicular to the magnetization vector $\mathbf{m}_{2}$ of the $\mathrm{F}_{2}$ layer. Therefore, it would be possible to determine the penetration depth of the transverse spin current in the $\mathrm{F}_{2}$ layer if we could analyze the dependence of the enhancement of the Gilbert damping constant on the thickness of the $\mathrm{F}_{2}$ layer.

In this letter, we study spin pumping in $\mathrm{N}_{1} / \mathrm{F}_{1} / \mathrm{N}_{2} /$ $\mathrm{F}_{2} / \mathrm{N}_{3}$ five-layer systems shown in Fig. 1 both theoretically and experimentally. We extend Tserkovnyak's theory of spin pumping by taking into account the finite penetration depth of the transverse spin current and show that the enhancement of the Gilbert damping con- 
stant due to spin pumping depends on the ratio of the penetration depth $\lambda_{\mathrm{t}}$ and the thickness $d_{2}$ of the $\mathrm{F}_{2}$ layer. The motion of the magnetization vector in the $\mathrm{F}_{2}$ layer is not in resonance with an oscillating magnetic field; hence, the $\mathrm{F}_{2}$ layer plays the role of spin absorber. We also perform FMR experiments in $\mathrm{Cu} / \mathrm{CoFe} / \mathrm{Cu} / \mathrm{Py} / \mathrm{Cu}$, $\mathrm{Cu} / \mathrm{Py} / \mathrm{Cu} / \mathrm{CoFe} / \mathrm{Cu}$ and $\mathrm{Cu} / \mathrm{CoFe} / \mathrm{Cu} / \mathrm{CoFeB} / \mathrm{Cu}$ fivelayer systems and measure line widths $\Delta B$ of energy absorption spectra, which are closely related to the Gilbert damping constants. The abbreviations $\mathrm{CoFe}, \mathrm{CoFeB}$, and $\mathrm{Py}$ hereafter refer to $\mathrm{Co}_{75} \mathrm{Fe}_{25},\left(\mathrm{Co}_{50} \mathrm{Fe}_{50}\right)_{80} \mathrm{~B}_{20}$ and $\mathrm{Ni}_{80} \mathrm{Fe}_{20}$, respectively. Analyzing the dependence of the line width on the thickness of the $\mathrm{Py}, \mathrm{CoFe}$ and $\mathrm{CoFeB}$ layers that are not in resonance, we showed that the penetration depths of the transverse spin current in the Py, CoFe and $\mathrm{CoFeB}$ layers are $3.7[\mathrm{~nm}], 2.5[\mathrm{~nm}]$ and 12.0 [nm], respectively.

Let us begin with an introduction to the theory of spin pumping in the nonmagnetic/ferromagnetic metal fivelayer system shown in Fig. 1 with a finite penetration depth of the transverse spin current. The pumped spin current generated by precession of the magnetization $\mathbf{m}_{1}$ of the $\mathrm{F}_{1}$ layer is given by

$$
\mathbf{I}_{s}^{\text {pump }}=\frac{\hbar}{4 \pi}\left(g_{r\left(\mathrm{~F}_{1}\right)}^{\uparrow \downarrow} \mathbf{m}_{1} \times \frac{\mathrm{d} \mathbf{m}_{1}}{\mathrm{~d} t}+g_{i\left(\mathrm{~F}_{1}\right)}^{\uparrow \downarrow} \frac{\mathrm{d} \mathbf{m}_{1}}{\mathrm{~d} t}\right),
$$

where $\hbar$ is the Dirac constant and $g_{r(i)}^{\uparrow \downarrow}$ is the real (imaginary) part of the mixing conductance [10]. The pumped spin current creates spin accumulation in the other layers, and the spin accumulation induces a backflow of spin current across each N/F interface. Although the backflow is obtained from circuit theory [6, 10], the penetration depth of the transverse spin current $\lambda_{\mathrm{t}}$ is assumed to be zero in this theory. Since we are interested in the effect of the penetration depth of the transverse spin current on spin pumping, we explicitly consider the diffusion process of transverse spin accumulation in the ferromagnetic layer. The backflow of spin current flowing from the $\mathrm{N}_{i}$ layer to the $\mathrm{F}_{k}$ layer is expressed as

$$
\begin{aligned}
& \mathbf{I}_{s}^{\mathrm{N}_{i} \rightarrow \mathrm{F}_{k}}=\frac{1}{4 \pi}\left[\frac{2 g^{\uparrow \uparrow} g^{\downarrow \downarrow}}{g^{\uparrow \uparrow}+g^{\downarrow \downarrow}}\left\{\mathbf{m}_{k} \cdot\left(\boldsymbol{\mu}_{\mathrm{N}_{i}}-\boldsymbol{\mu}_{\mathrm{F}_{k}}^{\mathrm{L}}\right)\right\} \mathbf{m}_{k}\right. \\
& +g_{r\left(\mathrm{~F}_{k}\right)}^{\uparrow \downarrow} \mathbf{m}_{k} \times\left(\boldsymbol{\mu}_{\mathrm{N}_{i}} \times \mathbf{m}_{k}\right)+g_{i\left(\mathrm{~F}_{k}\right)}^{\uparrow \downarrow} \boldsymbol{\mu}_{\mathrm{N}_{i}} \times \mathbf{m}_{k} \\
& \left.-t_{r\left(\mathrm{~F}_{k}\right)}^{\uparrow \downarrow} \mathbf{m}_{k} \times\left(\boldsymbol{\mu}_{\mathrm{F}_{k}}^{\mathrm{T}} \times \mathbf{m}_{k}\right)-t_{i\left(\mathrm{~F}_{k}\right)}^{\uparrow \downarrow} \boldsymbol{\mu}_{\mathrm{F}_{k}}^{\mathrm{T}} \times \mathbf{m}_{k}\right]
\end{aligned}
$$

where $g^{\uparrow \uparrow(\downarrow \downarrow)}$ is the spin up (down) conductance, $t_{r(i)}^{\uparrow \downarrow}$ is the real (imaginary) part of the transmission mixing conductance at the $\mathrm{F}_{k} / \mathrm{N}_{i}$ interface and $\boldsymbol{\mu}_{\mathrm{N}_{i}}$ is the spin accumulation in the $\mathrm{N}_{i}$ layer [6]. The longitudinal spin accumulation in the $\mathrm{F}_{k}$ layer is denoted by $\boldsymbol{\mu}_{\mathrm{F}_{k}}^{\mathrm{L}}$. The last two terms express contributions from transverse spin accumulation $\boldsymbol{\mu}_{\mathrm{F}_{k}}^{\mathrm{T}}$ in the $\mathrm{F}_{k}$ layer.

The spin accumulation in a ferromagnetic layer is defined by the non-equilibrium distribution matrix at a given energy $\varepsilon, \hat{f}(\varepsilon)=f_{0} \hat{1}+\boldsymbol{f} \cdot \hat{\boldsymbol{\sigma}}[\underline{6}]$, where $\boldsymbol{f}=$ $f_{x} \mathbf{t}_{1}+f_{y} \mathbf{t}_{2}+f_{z} \mathbf{m}$. Here we introduce the orthogonal unit vectors in spin space $\left(\mathbf{t}_{1}, \mathbf{t}_{2}, \mathbf{m}\right)$. The non-equilibrium charge distribution is represented by $f_{0}=\left(f^{\uparrow}+f^{\downarrow}\right) / 2$. On the other hand, $f_{z}=\left(f^{\uparrow}-f^{\downarrow}\right) / 2$ is the difference in non-equilibrium distribution between spin-up and spindown electrons, and $f_{x}$ and $f_{y}$ are the non-equilibrium distributions of the transverse spin components. The spin accumulation is defined as $\boldsymbol{\mu}=\int_{\varepsilon_{\mathrm{F}}} \mathrm{d} \varepsilon \operatorname{Tr}[\hat{\boldsymbol{\sigma}} \hat{f}]$ [10]. The spin accumulation in the nonmagnetic layer is defined in a similar way.

The spin accumulation in a nonmagnetic layer, $\boldsymbol{\mu}_{\mathrm{N}}$, obeys the diffusion equation [11], and is expressed as a linear combination of $\exp \left( \pm x / \lambda_{\mathrm{sd}(\mathrm{N})}\right)$, where $\lambda_{\mathrm{sd}(\mathrm{N})}$ is the spin diffusion length. The spin current in a nonmagnetic layer is given by

$$
\mathbf{I}_{s}^{\mathrm{N}}=-\frac{\partial}{\partial x} \frac{\hbar S \sigma_{\mathrm{N}}}{2 e^{2}} \boldsymbol{\mu}_{\mathrm{N}}
$$

where $S$ is the cross section area of the system, $\sigma_{\mathrm{N}}$ is the conductivity and $e$ is the absolute value of the electron charge. The spin current in the $\mathrm{N}_{3}$ layer is equal to $-\mathbf{I}_{s}^{\mathrm{N}_{3} \rightarrow \mathrm{F}_{2}}$ at $x=L_{2}+d_{2}$ because of the continuity of the spin current, and vanishes at $x=L_{2}+d_{2}+L_{3}$ (see Fig. (1). Using above boundary conditions, we obtain the spin accumulation in the $\mathrm{N}_{3}$ layer.

The longitudinal spin current in a ferromagnetic layer is given by

$$
\left(\mathbf{m} \cdot \mathbf{I}_{s}^{\mathrm{F}}\right) \mathbf{m}=-\frac{\partial}{\partial x} \frac{\hbar S}{2 e^{2}}\left(\sigma^{\uparrow} \mu_{\mathrm{F}}^{\uparrow}-\sigma^{\downarrow} \mu_{\mathrm{F}}^{\downarrow}\right) \mathbf{m},
$$

where $\mu_{\mathrm{F}}^{\uparrow(\downarrow)}=\int_{\varepsilon_{\mathrm{F}}} \mathrm{d} \varepsilon f^{\uparrow(\downarrow)}$ is the electro-chemical potential for the spin-up (spin-down) electrons and $\sigma^{\uparrow(\downarrow)}$ is the conductivity of spin-up (spin-down) electrons. The polarization of spin-dependent conductivity is defined as $\beta=\left(\sigma^{\uparrow}-\sigma^{\downarrow}\right) /\left(\sigma^{\uparrow}+\sigma^{\downarrow}\right)$. The longitudinal spin current in the $\mathrm{F}_{2}$ layer is equal to $\mathbf{m}_{2} \cdot \mathbf{I}_{s}^{\mathrm{N}_{2} \rightarrow \mathrm{F}_{2}}$ at $x=L_{2}$ and $-\mathbf{m}_{2} \cdot \mathbf{I}_{s}^{\mathrm{N}_{3} \rightarrow \mathrm{F}_{2}}$ at $x=L_{2}+d_{2}$ because of the continuity of the spin current. Solving the diffusion equation [11] with the above boundary conditions, we obtain longitudinal spin accumulation in the $\mathrm{F}_{2}$ layer. The longitudinal spin accumulation is expressed as a linear combination of $\exp \left( \pm x / \lambda_{\mathrm{sd}\left(\mathrm{F}_{\mathrm{L}}\right)}\right)$, where $\lambda_{\mathrm{sd}\left(\mathrm{F}_{\mathrm{L}}\right)}$ is the longitudinal spin diffusion length.

We assume that the transverse spin accumulation obeys the following equation [7]:

$$
\frac{\partial^{2}}{\partial x^{2}} \boldsymbol{\mu}_{\mathrm{F}}^{\mathrm{T}}=\frac{1}{\lambda_{J}^{2}} \boldsymbol{\mu}_{\mathrm{F}}^{\mathrm{T}} \times \mathbf{m}+\frac{1}{\lambda_{\mathrm{sd}\left(\mathrm{F}_{\mathrm{T}}\right)}^{2}} \boldsymbol{\mu}_{\mathrm{F}}^{\mathrm{T}},
$$

where $\lambda_{J}=\sqrt{\left(D^{\uparrow}+D^{\downarrow}\right) \hbar /(2 J)}$ and $\lambda_{\mathrm{sd}\left(\mathrm{F}_{\mathrm{T}}\right)}$ is the transverse spin diffusion length. Here $J$ represents the strength of the exchange field. The transverse spin accumulation is expressed as a linear combination of $\exp \left( \pm x / l_{+}\right)$and $\exp \left( \pm x / l_{-}\right)$, where $1 / l_{ \pm}=$ $\sqrt{\left(1 / \lambda_{\mathrm{sd}\left(\mathrm{F}^{\mathrm{T}}\right)}^{2}\right) \mp\left(\mathrm{i} / \lambda_{J}^{2}\right)}$. Therefore, we define the penetration depth of the transverse spin current $\lambda_{\mathrm{t}}$ by

$$
\frac{1}{\lambda_{\mathrm{t}}}=\operatorname{Re}\left[\frac{1}{l_{+}}\right] \text {. }
$$


The transverse spin current in a ferromagnetic layer is expressed as

$$
\mathbf{m} \times\left(\mathbf{I}_{s}^{\mathrm{F}} \times \mathbf{m}\right)=-\frac{\partial}{\partial x} \frac{\hbar S \sigma^{\uparrow \downarrow}}{2 e^{2}} \boldsymbol{\mu}_{\mathrm{F}}^{\mathrm{T}},
$$

where $\sigma^{\uparrow \downarrow}=(1 / 2)\left(\sigma^{\uparrow} /\left(1+\beta^{\prime}\right)+\sigma^{\downarrow} /\left(1-\beta^{\prime}\right)\right)$. Here $\beta^{\prime}=\left(D^{\uparrow}-D^{\downarrow}\right) /\left(D^{\uparrow}+D^{\downarrow}\right)$ is the polarization of the spin-dependent diffusion constants, $D^{\uparrow}$ and $D^{\downarrow}$ [7]. For simiplicty, we assume that $\beta=\beta^{\prime}$. The transverse spin accumulation in a ferromagnetic layer is obtained by solving Eq. (5) with boundary conditions satisfying the continuity of the spin current at the N/F interface.

Solving the diffusion equations of the spin accumulations of the $\mathrm{N}_{3}$ and $\mathrm{F}_{2}$ layers, the backflow at the $\mathrm{N}_{2} / \mathrm{F}_{2}$ interface is re-written as

$$
\begin{aligned}
\mathbf{I}_{s}^{\mathrm{N}_{2} \rightarrow \mathrm{F}_{2}}=\frac{1}{4 \pi} & {\left[g_{\left(\mathrm{F}_{2}\right)}^{*}\left(\mathbf{m}_{2} \cdot \boldsymbol{\mu}_{\mathrm{N}_{2}}\right) \mathbf{m}_{2}\right.} \\
& \left.+\tilde{g}_{r\left(\mathrm{~F}_{2}\right)}^{\uparrow \downarrow} \mathbf{m}_{2} \times\left(\boldsymbol{\mu}_{\mathrm{N}_{2}} \times \mathbf{m}_{2}\right)+\tilde{g}_{i\left(\mathrm{~F}_{2}\right)}^{\uparrow \downarrow} \boldsymbol{\mu}_{\mathrm{N}_{2}} \times \mathbf{m}_{2}\right],
\end{aligned}
$$

where the conductance $g_{\left(\mathrm{F}_{2}\right)}^{*}$ is given in Ref. [10], and depends on the ratio $d_{2} / \lambda_{\mathrm{sd}\left(\mathrm{F}_{\mathrm{L}}\right)}$. Similarly, the renormalized mixing conductances, $\tilde{g}_{r, i\left(\mathrm{~F}_{2}\right)}^{\uparrow \downarrow}$, depend on the ratio $d_{2} / l_{+\left(\mathrm{F}_{2}\right)}$. If the thickness of the $\mathrm{N}_{3}$ layer is thin enough compared to its spin diffusion length, $\tilde{g}_{r, i\left(\mathrm{~F}_{2}\right)}^{\uparrow \downarrow}$ are given by

$$
\left(\begin{array}{l}
\tilde{g}_{r\left(\mathrm{~F}_{2}\right)}^{\uparrow \downarrow} \\
\tilde{g}_{i\left(\mathrm{~F}_{2}\right)}^{\uparrow \downarrow}
\end{array}\right)=\frac{1}{\Delta}\left(\begin{array}{cc}
K_{1} & K_{2} \\
-K_{2} & K_{1}
\end{array}\right)\left(\begin{array}{c}
g_{r r\left(\mathrm{~F}_{2}\right)}^{\uparrow \downarrow} \\
g_{i\left(\mathrm{~F}_{2}\right)}^{\uparrow \downarrow}
\end{array}\right),
$$

where $\Delta=K_{1}^{2}+K_{2}^{2}$ and $K_{1}$ and $K_{2}$ are given

$$
\begin{aligned}
K_{1}=1 & +t_{r\left(\mathrm{~F}_{2}\right)}^{\uparrow \downarrow} \operatorname{Re}\left[\frac{1}{g_{\mathrm{t}} \tanh \left(d_{2} / l_{+}\right)}\right] \\
& +t_{i\left(\mathrm{~F}_{2}\right)}^{\uparrow \downarrow} \operatorname{Im}\left[\frac{1}{g_{\mathrm{t}} \tanh \left(d_{2} / l_{+}\right)}\right],
\end{aligned}
$$

$K_{2}=t_{i\left(\mathrm{~F}_{2}\right)}^{\uparrow \downarrow} \operatorname{Re}\left[\frac{1}{g_{\mathrm{t}} \tanh \left(d_{2} / l_{+}\right)}\right]-t_{r\left(\mathrm{~F}_{2}\right)}^{\uparrow \downarrow} \operatorname{Im}\left[\frac{1}{g_{\mathrm{t}} \tanh \left(d_{2} / l_{+}\right)}\right]$,

where $g_{\mathrm{t}} / S=h / 2 e^{2} \rho_{\mathrm{F}_{2}} l_{+}$and $\rho_{\mathrm{F}_{2}}$ is the resistivity of the $\mathrm{F}_{2}$ layer. The mixing conductance of the $\mathrm{F}_{1}$ layer in Eqs. (1) and (2) is also replaced by the renormalized conductance.

We assume that spin-flip scattering in the $\mathrm{N}_{2}$ layer is so weak that we can neglect the spatial variation of the spin current in the $\mathrm{N}_{2}$ layer. Then we have $\mathbf{I}_{s}^{\text {pump }}-\mathbf{I}_{s}^{\mathrm{N}_{1} \rightarrow \mathrm{F}_{1}}=\mathbf{I}_{s}^{\mathrm{N}_{2} \rightarrow \mathrm{F}_{2}}$, and the spin accumulation in the $\mathrm{N}_{2}$ layer can be determined [10]. The torque acting on the magnetization of the $\mathrm{F}_{1}$ layer is given by $\mathbf{m}_{1} \times\left\{\left(\mathbf{I}_{s}^{\text {pump }}-\mathbf{I}_{s}^{\mathrm{N}_{2} \rightarrow \mathrm{F}_{1}}\right) \times \mathbf{m}_{1}\right\}$, which yields the following modified Landau-Lifshitz-Gilbert (LLG) equation [10, 12] :

$$
\frac{\mathrm{d} \mathbf{m}_{1}}{\mathrm{~d} t}=-\gamma_{\mathrm{eff}} \mathbf{m}_{1} \times \mathbf{B}_{\mathrm{eff}}+\frac{\gamma_{\mathrm{eff}}}{\gamma}\left(\alpha_{0}+\alpha^{\prime}\right) \mathbf{m}_{1} \times \frac{\mathrm{d} \mathbf{m}_{1}}{\mathrm{~d} t},
$$
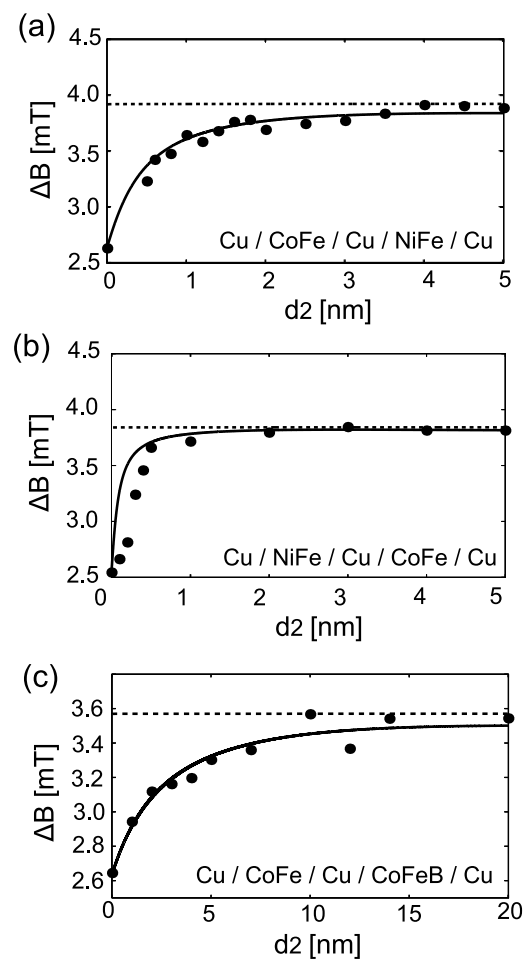

FIG. 2: The dependences of the line width of the FMR power absorption spectra, $\Delta B$, on the thickness of the $\mathrm{F}_{2}$ layer, $d_{2}$. Materials of the $\mathrm{F}_{2}$ layer are (a) $\mathrm{Ni}_{80} \mathrm{Fe}_{20}$, (b) $\mathrm{Co}_{75} \mathrm{Fe}_{25}$ and (c) $\left(\mathrm{Co}_{50} \mathrm{~F}_{50}\right)_{80} \mathrm{~B}_{20}$, respectively. The filled circles represent experimental data and the solid lines are fit to the experimental data according to the theory with the finite penetration depth of the transverse spin current $\lambda_{t}$. The dotted lines represent the case of $\lambda_{\mathrm{t}}=0$.

where $\mathbf{B}_{\text {eff }}$ is the effective magnetic field, $\gamma$ is the gyromagnetic ratio, $\alpha_{0}$ is the Gilbert damping constant intrinsic to the ferromagnetic metal, and $\alpha^{\prime}$ is the enhancement of the Gilbert damping constant due to spin pumping. The Gilbert damping constant is related to the line width of the FMR absorption spectrum via [13]

$$
\Delta B=\Delta B_{0}+\frac{2 \omega}{\sqrt{3} \gamma} \alpha^{\prime}
$$

where $\omega=2 \pi f$ is the angular velocity of the oscillating magnetic field. We notice that the effects of the $N_{1}$ and $\mathrm{N}_{3}$ layers are quite small because, as mentioned below, the thickness of these layers are thin enough compared to its spin diffusion length in our experiments. Assuming that $g_{r}^{\uparrow \downarrow} \gg g_{i}^{\uparrow \downarrow}$ [10], in the limit of $\theta \rightarrow 0$, we find

$$
\Delta B-\Delta B_{0} \simeq \frac{\hbar \omega}{2 \sqrt{3} \pi M d_{1} S} \frac{\tilde{g}_{r\left(\mathrm{~F}_{1}\right)}^{\uparrow \downarrow} \tilde{g}_{r\left(\mathrm{~F}_{2}\right)}^{\uparrow \downarrow}}{\tilde{g}_{r\left(\mathrm{~F}_{1}\right)}^{\uparrow \downarrow}+\tilde{g}_{r\left(\mathrm{~F}_{2}\right)}^{\uparrow \downarrow}},
$$

where $\tilde{g}_{r\left(\mathrm{~F}_{i}\right)}^{\uparrow \downarrow}(i=1,2)$ is the real part of the renormalized mixing conductance of the $i$-th ferromagnetic layer. We should note that if we neglect the transverse spin accumulation in the ferromagnetic layer the mixing conductances are not renormalized, and that the line width 
$\Delta B$ does not depend on the thickness of the $\mathrm{F}_{2}$ layer [10]. This is due to the fact that the dominant component of the pumped spin current is perpendicular to the magnetization vector $\mathbf{m}_{2}$ of the $\mathrm{F}_{2}$ layer in our experiment.

We performed FMR experiments on the three different $\mathrm{N}_{1} / \mathrm{F}_{1} / \mathrm{N}_{2} / \mathrm{F}_{2} / \mathrm{N}_{3}$ five-layer systems shown in Fig. 1 [14]. Nonmagnetic layers are made of $\mathrm{Cu}$. The combinations of the ferromagnetic layers $\left(\mathrm{F}_{1}, \mathrm{~F}_{2}\right)$ of each system are (a) $(\mathrm{CoFe}, \mathrm{Py}),(\mathrm{b})(\mathrm{Py}, \mathrm{CoFe})$ and (c) $(\mathrm{CoFe}, \mathrm{CoFeB})$. The samples were deposited on Corning 1737 glass substrates using an rf magnetron sputtering system in an ultrahigh vacuum below $4 \times 10^{-6}[\mathrm{~Pa}]$ and cut to $5\left[\mathrm{~mm}^{2}\right]$. The Ar pressure during deposition was $0.077[\mathrm{~Pa}]$. The thickness of all $\mathrm{Cu}$ layers are $5[\mathrm{~nm}]$. The thickness of $\mathrm{F}_{1}$ layers is $5[\mathrm{~nm}]$ for sample (a) and (b), and $10[\mathrm{~nm}]$ for sample (c). The FMR measurements were carried out using an X-band microwave source $(f=9.4[\mathrm{GHz}])$ at room temperature. The microwave power, modulation frequency, and modulation field are $1[\mathrm{~mW}], 10[\mathrm{kHz}]$, and $0.1[\mathrm{mT}]$, respectively. The precession angles of all samples are estimated to be $1[\mathrm{deg}]$. The resistivity $\rho_{\mathrm{F}}$ of Py, CoFe and $\mathrm{CoFeB}$ are $241[\Omega \mathrm{nm}], 94[\Omega \mathrm{nm}]$ and $1252[\Omega \mathrm{nm}]$, respectively. The magnetizations $(4 \pi M)$ of Py and CoFe are $0.76[\mathrm{~T}]$ and $2.1[\mathrm{~T}]$, respectively. The gyromagnetic ratio is $1.8467 \times 10^{11}[\mathrm{~Hz} / \mathrm{T}]$ for all systems

In Figs. 2 (a), (b) and (c), the measured line widths of the FMR absorption spectra $\Delta B$ are plotted with filled circles against the thickness of the $\mathrm{F}_{2}$ layer, $d_{2}$. The solid lines are fit to the experimental data according to the theory with the finite penetration depth of the transverse spin current $\lambda_{\mathrm{t}}$. The dotted lines represent the calculated $\Delta B$ in the case of $\lambda_{\mathrm{t}}=0$ [10].

Parameters other than $\lambda_{\mathrm{t}}$ are determined as follows. The mixing conductances per unit area of the combinations $\left(g_{r\left(\mathrm{~F}_{1}\right)}^{\uparrow \downarrow} / S, g_{r\left(\mathrm{~F}_{2}\right)}^{\uparrow \downarrow} / S\right)$ are assumed to be (a) (48.0, $38.0)$, (b) $(15.2,17.0)$ and (c) $(48.0,128.0)\left[\mathrm{nm}^{-2}\right]$. Although these values are determined by fitting, they have good agreement with the $a b$ initio caluclations [ $[6]$. For simplicity, we assume that $t_{r}^{\uparrow \downarrow}=t_{i}^{\uparrow \downarrow}$ where values of $t_{r, i}^{\uparrow \downarrow} / S$ of Py, CoFe and CoFeB are taken to be $4.0\left[\mathrm{~nm}^{-2}\right], 6.0$ $\left[\mathrm{nm}^{-2}\right]$ and $0.8\left[\mathrm{~nm}^{-2}\right]$, respectively. The longitudinal spin diffusion lengths are $5.5[\mathrm{~nm}]$ for Py and $12[\mathrm{~nm}]$ for $\mathrm{CoFe}$ and $\mathrm{CoFeB}$, respectively [15, 16]. The polarizations of conductance $\beta$ are 0.73 for $\mathrm{Py}, 0.65$ for $\mathrm{CoFe}$ and 0.56 for $\mathrm{CoFeB}$, respectively [15, 16, 17]. The transverse spin diffusion lengths are given by $\lambda_{\mathrm{sd}\left(\mathrm{F}_{\mathrm{T}}\right)}=\lambda_{\mathrm{sd}\left(\mathrm{F}_{\mathrm{L}}\right)} / \sqrt{1-\beta^{2}}$ 7]. We take $g_{i}^{\uparrow \downarrow} / S=1.0\left[\mathrm{~nm}^{-2}\right], 2 g^{\uparrow \uparrow} g^{\downarrow \downarrow} /\left(g^{\uparrow \uparrow}+g^{\downarrow \downarrow}\right) S=$ $20.0\left[\mathrm{~nm}^{-2}\right]$ 6, 10] for all systems; these are not important parameters for fitting the experimental results. The spin diffusion length and resistivity of $\mathrm{Cu}$ are taken to be $500[\mathrm{~nm}]$ and $21[\Omega \mathrm{nm}][18]$. The obtained values of $\lambda_{\mathrm{t}}$ are $3.7[\mathrm{~nm}]$ for Py, $2.5[\mathrm{~nm}]$ for CoFe and $12.0[\mathrm{~nm}]$ for $\mathrm{CoFeB}$, respectively. Our results agree quite well with the prediction based on the Boltzmann theory of electron transport [7].

In conclusion, we analyzed spin pumping in $\mathrm{Cu} / \mathrm{CoFe} / \mathrm{Cu} / \mathrm{Py} / \mathrm{Cu}, \quad \mathrm{Cu} / \mathrm{Py} / \mathrm{Cu} / \mathrm{CoFe} / \mathrm{Cu}$ and $\mathrm{Cu} / \mathrm{CoFe} / \mathrm{Cu} / \mathrm{CoFeB} / \mathrm{Cu}$ five-layer systems both theoretically and experimentally. We showed that the enhancement of the Gilbert dumping constant due to spin pumping depends on the ratio of the penetration depth $\lambda_{\mathrm{t}}$ and the thickness of the ferromagnetic layers, which is not in resonance with the oscillating magnetic field. We measured the line widths of FMR absorption spectra, which are closely related to the Gilbert dumping constant. Analyzing the experimental results, we showed that the penetration depths of the transverse spin current in $\mathrm{Py}, \mathrm{CoFe}$ and $\mathrm{CoFeB}$ are $3.7[\mathrm{~nm}], 2.5$ $[\mathrm{nm}]$ and $12.0[\mathrm{~nm}]$, respectively. Our results support the Boltzmann theory of transverse spin current [7].

The authors would like to acknowledge the valuable discussions they had with P. M. Levy. This work was supported by CREST and NEDO.
[1] J. C. Slonczewski, J. Magn. Magn. Mater. 159, L1 (1996).

[2] L. Berger, Phys. Rev. B 54, 9353 (1996).

[3] J. Z. Sun, Phys. Rev. B 62, 570 (2000).

[4] S. I. Kiselev, J. C. Sankey, I. N. Krivorotov, N. C. Emley, R. J. Schoelkopf, R. A. Buhrman, and D. C. Ralph, Nature 425, 380 (2003).

[5] M. D. Stiles and A. Zangwill, Phys. Rev. B 66, 014407 (2002).

[6] A. Brataas, G. E. W. Bauer, and P. J. Kelly, Phys. Rep. 427, 157 (2006).

[7] S. Zhang, P. M. Levy, and A. Fert, Phys. Rev. Lett. 88, 236601 (2002).

[8] S. Urazhdin, R. Loloee, and W. P. Pratt, Jr, Phys. Rev. B 71, 100401(R) (2005).

[9] W. Chen, M. J. Rooks, N. Ruiz, J. Z. Sun, and A. D. Kent, Phys. Rev. B 74, 144408 (2006).

[10] Y. Tserkovnyak, A. Brataas, G. E. W. Bauer, and B. I. Halperin, Rev. Mod. Phys. 77, 1375 (2005).
[11] T. Valet and A. Fert, Phys. Rev. B 48, 7099 (1993).

[12] T. Taniguchi and H. Imamura, Phys. Rev. B 76, 092402 (2007).

[13] S. V. Vonsovskii, FERROMAGNETIC RESONANCE (Israel Program for Scientific Translations Ltd., Jersalem, 1964).

[14] S. Mizukami, Y. Ando, and T. Miyazaki, J. Magn. Magn. Mater. 226, 1640 (2001); Jpn. J. Appl. Phys. 1 40, 580 (2001); Phys. Rev. B 66, 104413 (2002); J. Magn. Magn. Mater. 239, 42 (2002).

[15] A. C. Reilly, W. Park, R. Slater, B. Ouaglal, R. Loloee, W. P. P. Jr., and J. Bass, J. Magn. Magn. Mater. 195, L269 (1999).

[16] A. Fert and L. Piraux, J. Magn. Magn. Mater. 200, 338 (1999).

[17] H. Oshima, K. Nagasaka, Y. Seyama, Y. Shimizu, S. Eguchi, and A. Tanaka, J. Appl. Phys. 91, 8105 (2002).

[18] J.Bass and W. P. P. Jr, J. Phys.: Condens. Matter 19, 183201 (2007). 